This document is confidential and is proprietary to the American Chemical Society and its authors. Do not copy or disclose without written permission. If you have received this item in error, notify the sender and delete all copies.

\title{
Anti-cancer Polymers via the Biginelli Reaction
}

\begin{tabular}{|r|l|}
\hline Journal: & ACS Macro Letters \\
\hline Manuscript ID & mz-2020-004963.R1 \\
\hline Manuscript Type: & Letter \\
\hline Date Submitted by the & 09-Aug-2020 \\
\hline Complete List of Authors: & $\begin{array}{l}\text { Li, Yongsan; Tsinghua University, Chemistry Department } \\
\text { Tan, Tianhao; Tsinghua University, Chemistry Department } \\
\text { zhao, yuan; Tsinghua University, Department of Chemistry } \\
\text { Wei, Yen; Tsinghua University, } \\
\text { Wang, Dong; Tsinghua University, Chemistry Department } \\
\text { Chen, Rongjun; Imperial College London, Department of Chemical } \\
\text { Engineering } \\
\text { Tao, Lei; Tsinghua University, Department of Chemistry }\end{array}$ \\
\hline
\end{tabular}

\section{SCHOLARONE" \\ Manuscripts}




\title{
Anti-cancer Polymers via the Biginelli Reaction
}

\author{
Yongsan Lia, Tianhao Tanb, Yuan Zhao ${ }^{a}$, Yen Weia, Dong Wangb*, Rongjun Chen* and Lei Tao ${ }^{a *}$ \\ a The Key Laboratory of Bioorganic Phosphorus Chemistry \& Chemical Biology (Ministry of Education), Department of \\ Chemistry, Tsinghua University, Beijing 100084, P. R. China. \\ ${ }^{b}$ MOE Key Laboratory of Organic OptoElectronics and Molecular Engineering, Department of Chemistry, Tsinghua \\ University, Beijing 100084, P. R. China. \\ c Department of Chemical Engineering, Imperial College London, South Kensington Campus, London, SW7 2AZ, U.K.
}

\begin{abstract}
We developed a polymer-drug strategy to explore anti-cancer polymers. A series of monomers containing groups with potential anti-cancer activity have been facilely prepared through the Biginelli reaction. These monomers were used to produce water-soluble polymers through convenient radical copolymerization. The resulting polymers are biocompatible and can be directly used to suppress proliferation of different cancer cells without the release of small molecules. Theoretical calculations revealed that Biginelli groups in polymers had strong interaction with the Eg5 protein, which is highly expressed in cancer cells and is closely related to cell mitosis. Subsequent cell experiments confirmed that screened polymer is efficient in inhibiting mitosis in different cancer cells. Our study of exploring functional polymers via the combination of multicomponent reactions and theoretical calculation resulted in promising anti-cancer polymers, which might pave a path for de novo designing of functional polymers and have important implications in the fields of organic, computational, and polymer chemistry.
\end{abstract}

In the past decades, we have observed the considerable successes of anti-cancer drugs. The best anti-cancer drugs, such as taxol and doxorubicin, save millions of patients every year ${ }^{1-3}$. However, severe side effects and poor bioavailability, which are mainly caused by poor water solubility, instability, and/or quick clearance rates from body of these small molecular drugs, greatly limit their therapeutic effects. To overcome the restrictions of small molecular drugs, in 1975, Ringsdorf proposed for the first time a milestone concept of polymer prodrug to improve safety, stability, solubility, and metabolic half-life of small molecular drugs using polymers ${ }^{4-5}$. Basically, a polymer prodrug consists of a water-soluble polymer, small molecular drugs, and degradable linkers; drugs gradually release from polymer prodrugs after the degradation of linkers under physiological conditions (Scheme 1a).

This polymer prodrug strategy spawned many elaborate polymer-drug conjugates ${ }^{6-9}$; however, it often experiences two problems: 1) it is complicated to design biodegradable linkers such as peptides and polysaccharides that can be smoothly cleaved by enzymes during metabolic processes; 2) multi-step reactions, including protection-deprotection approaches, are necessary to link small molecular drugs on polymer chains. These complications limit the large-scale preparation and application of polymer prodrugs. Thus, the development of a new strategy to facilely prepare biocompatible polymers with superior anti-cancer activity is essential for academic studies and practical applications.

Scheme 1. a) Polymer-prodrug strategy, and b) polymer-drug strategy via MCRs.

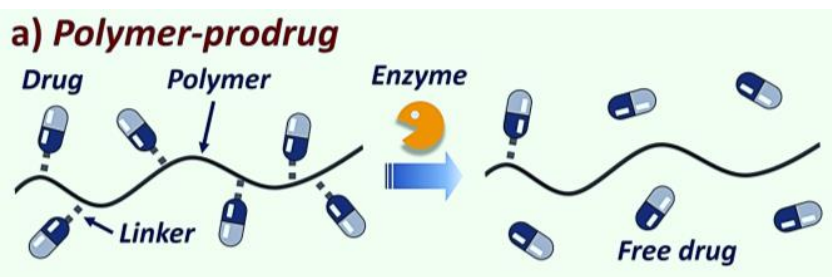

\section{b) Polymer-drug}

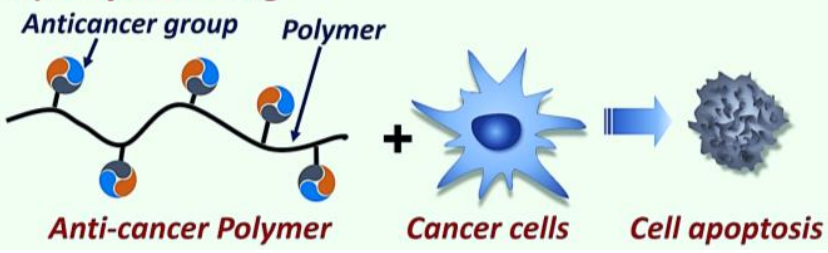

Recently, multicomponent reactions (MCRs) have been used in polymer chemistry. Many polymers with multicomponent main/side chains have been prepared by different MCRs such as Passerini, Ugi, Mannich, Biginelli, Hantzsch, and Kabachnik-Fields reactions ${ }^{10-28}$. These multicomponent groups confer interesting properties/functions to polymers. For example, the Biginelli reaction, which was introduced by Pietro Biginelli in $1891^{29}$, uses an aldehyde, a $\beta$ ketoester, and a (thio)urea to effectively produce dihydropyrimidones (DHPMs) ${ }^{30}$ (Scheme 2) that possess antibacterial, antitumor, and antioxidant bioactivities ${ }^{31}$.

Scheme 2. a) The Biginelli reaction, and b) a possible mechanism of the Biginelli reaction. 


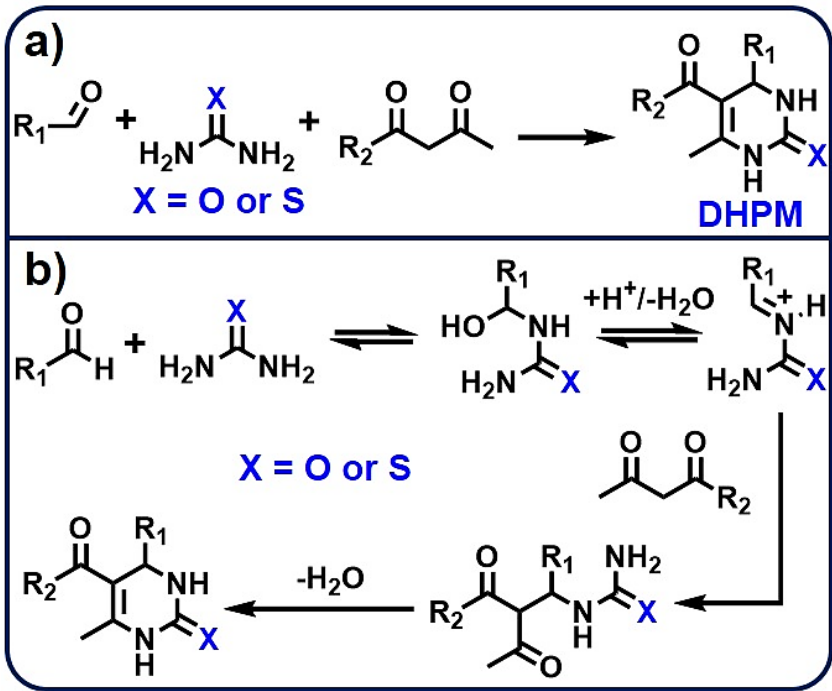

In recent studies, we used thiourea and its derivatives to prepare polymers containing DHPM side groups via the Biginelli reaction ${ }^{32-35}$. These polymers worked directly as excellent macromolecular antioxidants without releasing small molecular DHPM moieties. Some optimized polymers performed even better than superoxide dismutase in protecting cells from lethal UV damage, and silymarin (an active ingredient in clinically prescribed medicine) in counteracting oxidative stress-induced acute liver injury, respectively ${ }^{33-34}$. These results suggest that the preparation of polymers by MCRs is a straightforward strategy to explore bioactive polymers for biological and/or medical applications.

Inspired by previous research, we propose a facile strategy to develop anti-cancer polymers. Anti-cancer groups that can be generated from MCRs are included into polymer chains. These polymers can be directly used as polymerdrugs to selectively inhibit the growth of cancer cells (Scheme 1b).

In this study, the Biginelli reaction was taken as a typical example of MCRs to develop anti-cancer polymers. Monastrol (Figure 1a) is a promising anti-cancer agent. It specifically inhibits Eg5 kinesin protein that is highly expressed in many tumor cells and is closely related to the occurrence and development of tumors, which leads to spindle collapse and cell cycle arrest ${ }^{36}$. Monastrol can be easily prepared using $m$-hydroxy benzaldehyde through the Biginelli reaction. In this study, several monomers containing monastrol moieties were prepared (Figure 1a).

The phenol group in monastrol was directly modified to obtain M1. The ethyl group in monastrol was replaced by small tri-ethylene glycol (TEG) to obtain a methacrylate monomer (M2). M3 was prepared using a TEG spacer to link the phenol group in monastrol and methacrylic acid. M4 is an isomer of M3, which uses $p$-hydroxy benzaldehyde as a reactant for the Biginelli reaction. These monomers can be readily prepared on gram scale (see the preparation details in the Supplementary Information). The ${ }^{1} \mathrm{H}$ NMR spectra of monomers (Figures 1b-1e) clearly show the characteristic peaks of Biginelli cyclization products (PhCH: 5.08-5.19 ppm, NH: $10.30-10.39$ ppm, and 9.60-9.71 ppm). As a typical example, in the ${ }^{1} \mathrm{H}$ NMR spectrum of M1 (Figure $1 \mathrm{~b}$ ), the integral ratios between protons in the vinyl group and $\mathrm{PhCH}$ methine ( $\left.\mathrm{I}_{6.28} / \mathrm{I}_{5.91} / \mathrm{I}_{5.19}\right)$ are $1 / 1 / 1.01$, which is consistent with the theoretical value $(1 / 1 / 1)$. Similar results were obtained for other monomers (Figures $1 c-1 e$ ), which suggests the smooth preparation of desired monomers.

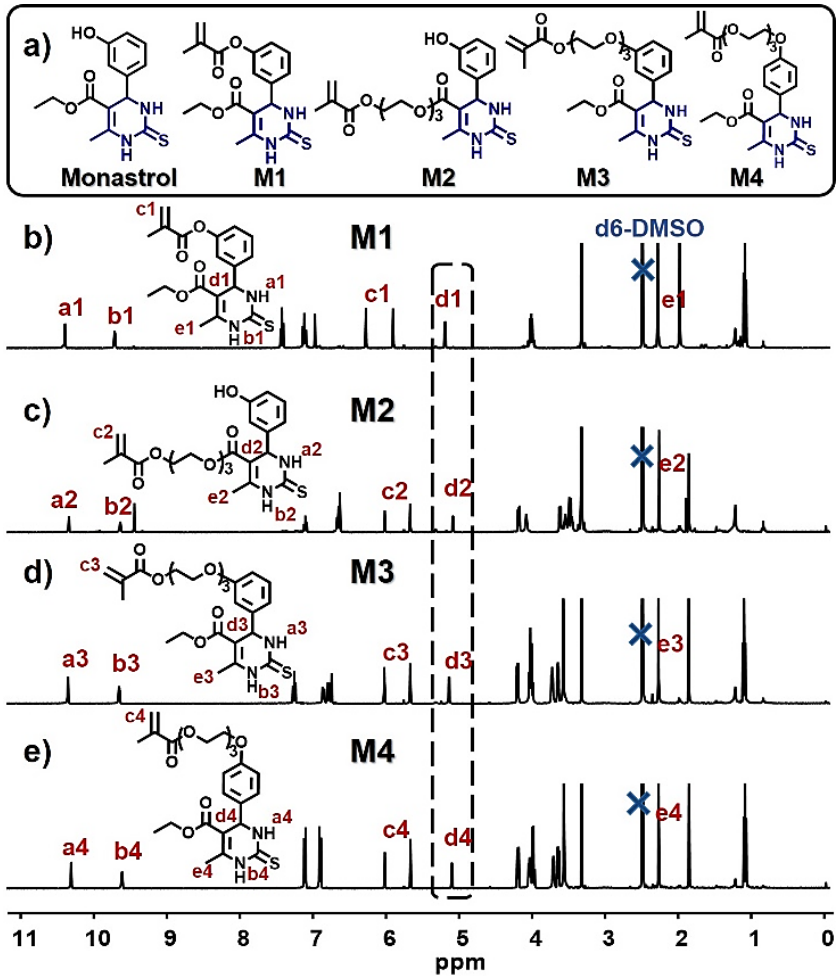

Figure 1. a) Monastrol and related monomers; b-e) ${ }^{1} \mathrm{H}$ NMR spectra (400 MHz, DMSO-d6) of the monomers: b) M1, c) M2, d) M3, and e) M4.

These monomers were copolymerized with a water-soluble monomer, poly(ethylene glycol methoxy ether) methacrylate (PEGMA, $\mathrm{M}_{\mathrm{n}} \sim 950 \mathrm{~g} \mathrm{~mol}^{-1}$ ) (molar ratio: $1 / 1$ ) to produce corresponding water-soluble polymers (P1, P2, P3, and $\mathrm{P} 4$ ) by convenient radical polymerization. All monomers were copolymerized with PEGMA with high conversion (96-98\%) (Figure S1, Table S1), suggesting the DPHM groups hardly affect radical polymerization. All polymers have satisfactory molecular weights $\left(\mathrm{M}_{\mathrm{n}}(\mathrm{GPC}): \sim 46200\right.$ $57300 \mathrm{~g} \mathrm{~mol}^{-1}$, Figure 2, Figure S2, and Table S1) and water solubility (> $150 \mathrm{mg} / \mathrm{mL}$, Table S1). The ${ }^{1} \mathrm{H}$ NMR spectra of polymers (Figure 2) show the characteristic peaks of Biginelli structures (5.10-5.18 ppm), and the methoxyl groups in PEG segments (3.23 ppm) are clearly visible. The integral ratios between the Biginelli structures and methoxyl groups at PEG chain ends ( $\left.\mathrm{I}_{5.09-5.18} / \mathrm{I}_{3.23}=0.97-1.01 / 3\right)$ agreed well with the theoretical value (1/3) suggesting these DHPM groups remained nearly intact during radical polymerization. These results indicate the smooth copolymerization of new monomers with PEGMA; the DHPM groups in monomers are compatible with radical polymerization, which is consistent with the results of previous studies using other monomers containing different DHPM groups for radical polymerization $^{33-34}$. 


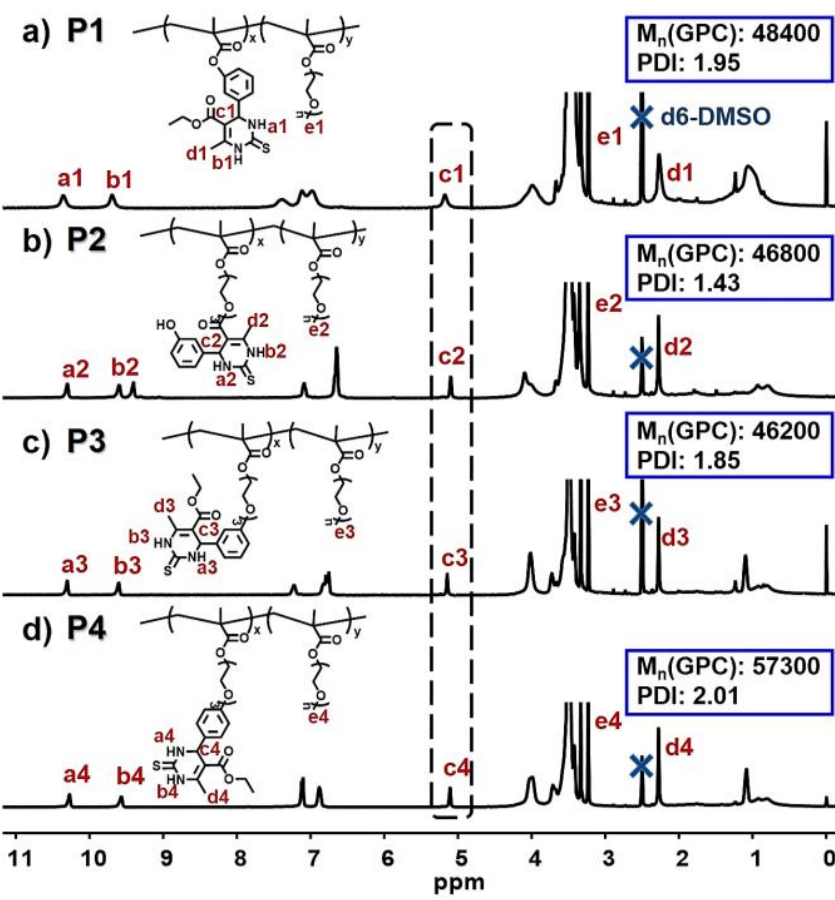

Figure 2. ${ }^{1} \mathrm{H}$ NMR spectra ( $400 \mathrm{MHz}$, DMSO- $d 6$ ) of polymers: a) P1; b) P2; c) P3; d) P4.

Biomaterials should be biocompatible. Thus, the cytotoxicity of these polymers was tested using a L929 cell line (a model of normal cells) through a CCK-8 assay (Figure 3a). A homopolymer of PEGMA was prepared by radical polymerization (P(PEG), Figure S3) and used as a control.

Most L929 cells ( $>90 \%$ ) survived the administration of $10 \mathrm{mg} / \mathrm{mL}$ polymers, which indicated good cytosafety of these Biginelli-type polymers (Figure 3a). When a human hepatoma cell line (SMMC-7721) was used (Figure 3b), P(PEG) exhibited almost no toxicity to SMMC-7721; however, the Biginelli-type polymers showed a clear concentration-dependent cytotoxicity to SMMC-7721 cells. This suggests that DHPM moieties in Biginelli-type polymers are essential for inhibiting the growth of SMMC-7721 cells. Similar results were observed when a human cervical cancer cell line (HeLa) and a human breast cancer cell line (MCF-7) were tested (Figure S4). These results demonstrate the selective anti-cancer capability of these Biginelli-type copolymers. At high concentrations ( $\geq 2.5 \mathrm{mg} / \mathrm{mL}$ ), these Biginellitype polymers followed a similar cytotoxicity order for three cancer cells: P4 > P3 > P2 $\approx$ P1 (Figure 3b, Figure S4). This result suggests that different DHPM pendent groups confer polymers different anti-cancer capabilities.

To understand the relationship between polymer structures and their anti-cancer ability, we performed molecular dynamics simulations using Gromacs Version 5.1.5. The DHPM moieties in polymer structures were simplified as units (Figure 3c). All polymers are made from monastrol and its derivatives; thus, we used the X-ray structure of Eg5 and monastrol (PDB ID: 1II6) as the starting structure, wherein monastrol is bound in a hydrophobic pocket formed by the $\alpha 2$-helix ( $\alpha 2$ ), $\alpha 3$-helix ( $\alpha 3$ ), and Loop 5 (L5) (Figure S5) to calculate the interaction energy between the Biginelli-type units and Eg5 kinesin protein (Figures $3 \mathrm{~d}$ and S6).
Unit-1 interacts only with the hydrophobic pocket $(\alpha 2$, $\alpha_{3}$ and L5) of Eg5 (Figure 3d1). The phenol group in unit-2 forms a hydrogen bond with Glu118 (Figure S6b); thus, unit-2 has stronger interaction energy than unit-1. However, the hydrophilic TEG group in unit-2 is almost completely exposed to the solvent $\left(\mathrm{H}_{2} \mathrm{O}\right)$ (Figure $3 \mathrm{~d} 2$ ) and has almost no contribution to stabilize the interaction of unit-2/Eg5. On the other hand, the TEG segments in unit-3 (linking on the meta phenol position) goes through L5 of Eg5 to strengthen the interaction between unit-3 and protein (Figures $3 \mathrm{~d} 3$ ). The TEG chain in unit-4 (linking on the para phenol position) is located in the hydrophobic groove between L5 and $\alpha 2$ (Figure 3d4); thus, a strong hydrogen bond is formed between $\operatorname{Arg} 138$ on the $\alpha 2$ helix and TEG chain (Figure S6d), which stabilizes the unit-4/protein complex. Thus, the calculated absolute interaction energy between different units and Eg5 is higher than that between monastrol and Eg5 (115 kJ/mol, Figure S5). The absolute values follow the order of unit-4 $(337 \mathrm{~kJ} / \mathrm{mol})>$ unit-3 $(324 \mathrm{~kJ} / \mathrm{mol})>$ unit-2 $(243 \mathrm{~kJ} / \mathrm{mol})>$ unit-1 $(206 \mathrm{~kJ} / \mathrm{mol})$ (Figure $3 \mathrm{~d})$, which agrees well with the anti-cancer ability of polymers obtained in the cell experiments. These results reveal the strong interaction between Biginelli-type polymers and Eg5 protein; thus, these polymers may, similar to monastrol, have the ability to inhibit mitosis in cancer cells.

To test this hypothesis, $\mathrm{P} 4$ at its safe concentration (viability of L929 cells $\geq 95 \%$, Figure $3 a$ ) $(5 \mathrm{mg} / \mathrm{mL})$ was selected as a typical example to study the anti-cancer mechanism of Biginelli-type polymers.

Fluorescein diacetate/propidium iodide (FDA/PI) double staining assay was performed to investigate the inhibition effect of P4 on different cells (Figures 4a and 4b). L929 cells cultured with $\mathrm{P} 4$ for 24 h have similar cell density as the control group (only cells in the medium), and dead cells (red spots) were not observed (Figure 4a). When SMMC7721 cells were used, dead cells were not detected; however, there were fewer cells cultured with P4 than those only in culture medium (Figure $4 \mathrm{~b}$ ). Similar results were observed when MCF-7 and HeLa cells were tested (Figure S7). These results suggest that $\mathrm{P} 4$ selectively inhibits the proliferation of cancer cells rather than killing them.

Furthermore, immunostaining was carried out to detect the inhibitory process of $\mathrm{P} 4$ during cellular proliferation. Cells were cultured with P4 for $24 \mathrm{~h}$, then stepwise incubated with primary antibody, FITC-tagged second antibody, and Hoechst 33342 to visualize the microtubules (green) and chromatin (blue). Cells in the culture medium served as controls (Figures 4c and 4d). 

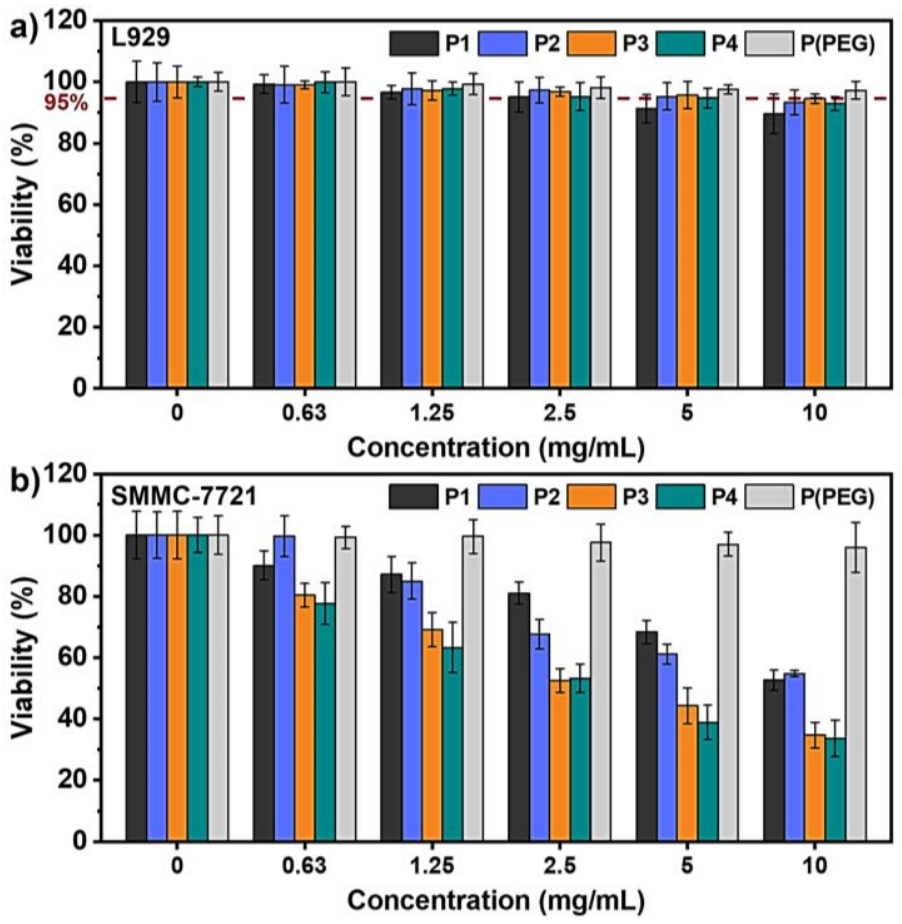

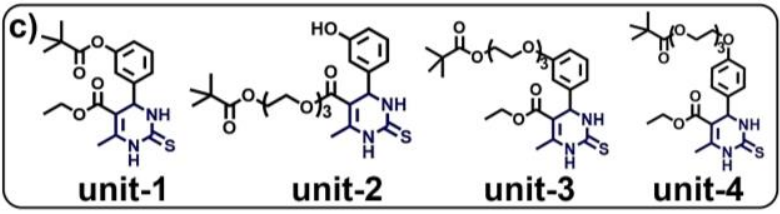

d1)
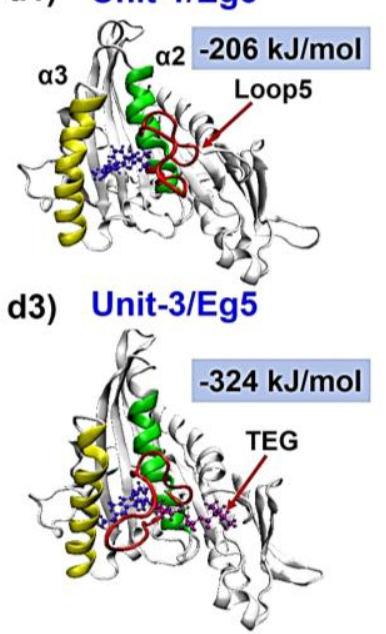

d2) Unit-2/Eg5
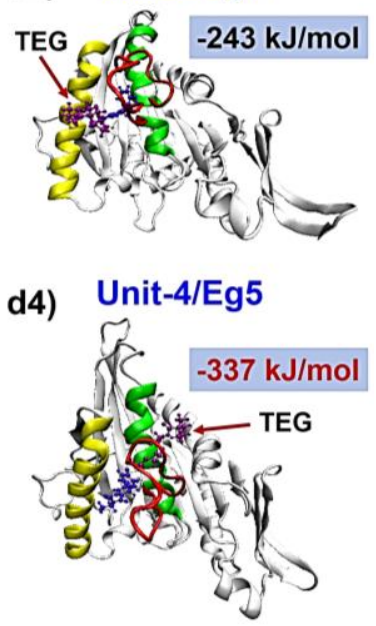

Figure 3. a, b) Cytotoxicity of polymers to L929 cells and SMMC-7721 cells, 48 h (a: L929 cells, b: SMMC-7721 cells), data are presented as mean $\pm S D, n=5$; c) simplified models of different polymers; d) simulated unit-Eg5 protein interaction and related interaction energy: d1) unit-1/Eg5, d2) unit-2/Eg5, d3) unit-3/Eg5, d4) unit-4/Eg5 (blue: DHPM groups, purple: TEG chains).

L929 cells cultured with P4 showed an ordinary mitosis like the control group (Figure 4c). However, in SMMC-7721 cells cultured with $\mathrm{P} 4$, chromatins did not move to the poles but were evenly radially distributed around the microtube protein (Figure 4d); thus, cells could not divide and proliferate. Similar results were observed for other cancer cells (MCF-7 and HeLa cells) (Figure S8), which suggested that P4 selectively inhibited mitosis in cancer cells. These results confirm that P4 shares a similar anti-cancer mechanism as small molecular monastrol; thus, it is a potential anti-cancer polymer.

Moreover, the stability of P4 under cell culture conditions was tested. P4 ( $5 \mathrm{mg} / \mathrm{mL}$ in culture medium) was kept in a metal shaker at $37^{\circ} \mathrm{C}$ for $48 \mathrm{~h}$. The solutions remained clear during the 48-h observation, which preliminary indicated that no obvious DHPM moiety was released from P4 (small molecular DHPMs have poor solubility in aqueous solutions). After $48 \mathrm{~h}$, this solution was lyophilized; P4 with possible generated small organic molecules were collected by extraction using $\mathrm{CH}_{2} \mathrm{Cl}_{2}$ followed by evaporation. The acquired sample was tested with GPC and ${ }^{1} \mathrm{H}$ NMR to assess the integrity of $\mathrm{P} 4$. No obvious change in the retention time was observed in GPC traces before and after the degradation test (Figure S9). In addition, no signs of a degraded small molecular DHPM moiety was detected by ${ }^{1} \mathrm{H}$ NMR (Figure S10). Similar results were also observed when other Biginelli-type polymers were tested except for $~ 3 \%$ hydrolyzation in P1 detected in the ${ }^{1} \mathrm{H}$ NMR spectrum (Figure S10). These results confirm the integrity of Biginelli polymers during cell experiments; thus, these polymer-drugs could directly act as polymeric anti-cancer agents without releasing small molecular therapeutics.
In summary, we developed a new strategy to prepare anti-cancer polymers via MCRs. A series of DHPM groups, which were similar to those in monastrol, were readily prepared through the Biginelli reaction (a typical example of MCRs) and included into monomers. These monomers were copolymerized with PEGMA to achieve water-soluble polymers. These polymers are biocompatible and can be used directly to efficiently inhibit the growth of different cancer cells. Theoretical calculations revealed that DHPM structures in polymers had strong interaction with an Eg5 kinesin protein; the anti-cancer activity of these DHPM groups can be effectively enhanced by the TEG group through suitable connection. In cell experiments, $\mathrm{P} 4$, a polymer with the best anti-cancer ability, effectively suppressed the proliferation of different cancer cells by inhibiting cell mitosis similar to monastrol, which confirmed the rationality of theoretical calculations.

Currently, MCRs are considered to be powerful tools for exploring new functional polymers. This study for the first time developed anti-cancer polymers via the Biginelli reaction, which may prompt a broad study of MCRs in polymer chemistry to create other new bioactive polymers through different MCRs. Meanwhile, the current research demonstrates that theoretical calculations are a powerful tool to understand the structure-property relationship of polymers, which provides basis for the optimization of polymer structures and may allow future de novo design of new functional polymers. 

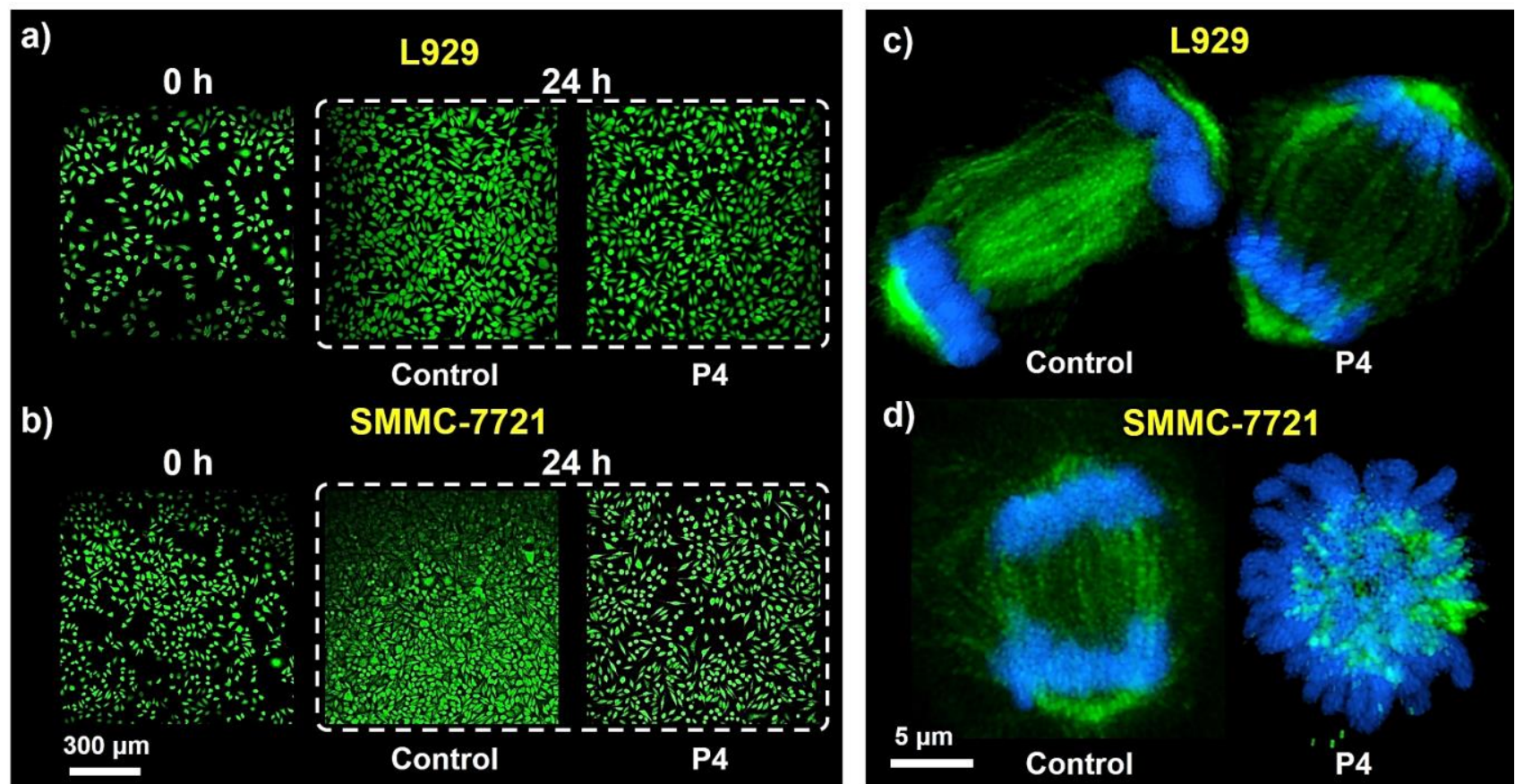

Figure 4. a, b) FDA/PI images of L929 and SMMC-7721 cells incubated with/without P4 (5 mg/mL), 24 h (a: L929 cells, b: SMMC7721 cells) (green: alive; red: dead); c, d) confocal images of L929 cells and SMMC-7721 cells incubated with/without P4 (5 $\mathrm{mg} / \mathrm{mL}$ ) using FITC-labeled anti-body/Hoechst 33342 for cell staining (green: microtube; blue: chromatin) (c: L929 cells, d: SMMC-7721 cells)

\section{ASSOCIATED CONTENT}

\section{Supporting Information}

The Supporting Information is available free of charge on the ACS Publications website. Syntheses and ${ }^{1} \mathrm{H}$ NMR, ${ }^{13} \mathrm{C}$ NMR, FTIR, ESI-MS data of the monomers, ${ }^{1} \mathrm{H}$ NMR, GPC data and anticancer tests of the polymers.

\section{AUTHOR INFORMATION}

\section{Corresponding Authors}

*E-mail: leitao@mail.tsinghua.edu.cn, dong913@mail.tsinghua.edu.cn, rongjun.chen@imperial.ac.uk.

\section{Notes}

The authors declare no conflict of interest.

\section{ACKNOWLEDGMENT}

This research was supported by the National Science Foundation of China $(21971141,21534006,21673123)$.

\section{REFERENCES}

1. Stage, T. B.; Bergmann, T. K.; Kroetz, D. L., Clinical Pharmacokinetics of Paclitaxel Monotherapy: An Updated Literature Review. Clin. Pharmacokinet. 2018, 57 (1), 7-19.

2. Alves, R. C.; Fernandes, R. P.; Eloy, J. O.; Salgado, H. R. N.; Chorilli, M., Characteristics, Properties and Analytical Methods of Paclitaxel: A Review. Crit. Rev. Anal. Chem. 2018, 48 (2), 110-118.

3. Bisogno, G.; Jenney, M.; Bergeron, C.; Gallego Melcón, S.; Ferrari, A.; Oberlin, 0.; Carli, M.; Stevens, M.; Kelsey, A.; De
Paoli, A.; Gaze, M. N.; Martelli, H.; Devalck, C.; Merks, J. H.; BenArush, M.; Glosli, H.; Chisholm, J.; Orbach, D.; Minard-Colin, V.; De Salvo, G. L.; Cesen, M.; Rose, A.; Ferman, S.; Mudry, P.; Sejnova, D.; Dal Bianco, P.; Zanetti, I.; Niggli, F.; Rogers, T.; Cecchetto, G.; De Corti, F.; Guerin, F.; Terwisscha, S.; Ranchere, D.; Alaggio, R.; Shipley, J.; Rosolen, A.; Mandeville, H.; Scarzello, G.; Bernier, V.; McHugh, K., Addition of dose-intensified doxorubicin to standard chemotherapy for rhabdomyosarcoma (EpSSG RMS 2005): a multicentre, openlabel, randomised controlled, phase 3 trial. Lancet Oncol. 2018, 19 (8), 1061-1071.

4. Ringsdorf, H., Structure and properties of pharmacologically active polymers. J. Polym. Sci., C Polym. Symp. 1975, 51 (1), 135-153.

5. Hoste, K.; De Winne, K.; Schacht, E., Polymeric prodrugs. Int. J. Pharm. 2004, 277 (1), 119-131.

6. Malugin, A.; Kopečková, P.; Kopeček, J., HPMA CopolymerBound Doxorubicin Induces Apoptosis in Human Ovarian Carcinoma Cells by a Fas-Independent Pathway. Mol. Pharm. 2004, 1 (3), 174-182.

7. Peng, Z.-H.; Kopeček, J., HPMA Copolymer CXCR4 Antagonist Conjugates Substantially Inhibited the Migration of Prostate Cancer Cells. ACS Macro Lett. 2014, 3 (12), 1240-1243.

8. Peng, Z.-H.; Kopeček, J., Enhancing Accumulation and Penetration of HPMA Copolymer-Doxorubicin Conjugates in 2D and 3D Prostate Cancer Cells via iRGD Conjugation with an MMP-2 Cleavable Spacer. J. Am. Chem. Soc. 2015, 137 (21), 6726-6729.

9. Ou, Y.; Chen, K.; Cai, H.; Zhang, H.; Gong, Q.; Wang, J.; Chen, W.; Luo, K., Enzyme/pH-sensitive polyHPMA-DOX conjugate as a biocompatible and efficient anticancer agent. Biomater. Sci. 2018, 6 (5), 1177-1188.

10. Kreye, O.; Toth, T.; Meier, M. A. R., Introducing Multicomponent Reactions to Polymer Science: Passerini Reactions of Renewable Monomers. J. Am. Chem. Soc. 2011, 133 (6), 1790-1792. 
11. Deng, X. X.; Li, L.; Li, Z. L.; Lv, A.; Du, F. S.; Li, Z. C., Sequence Regulated Poly(ester-amide)s Based on Passerini Reaction. ACS Macro Lett. 2012, 1 (11), 1300-1303.

12. Kreye, O.; Turunc, O.; Sehlinger, A.; Rackwitz, J.; Meier, M. A. R., Structurally Diverse Polyamides Obtained from Monomers Derived via the Ugi Multicomponent Reaction. Chem-Eur. J. 2012, 18 (18), 5767-5776.

13. Zhu, C. Y.; Yang, B.; Zhao, Y. A.; Fu, C. K.; Tao, L.; Wei, Y., A new insight into the Biginelli reaction: the dawn of multicomponent click chemistry? Polym. Chem. 2013, 4 (21), 5395-5400.

14. Solleder, S. C.; Meier, M. A. R., Sequence Control in Polymer Chemistry through the Passerini Three-Component Reaction. Angew. Chem. Int. Edit. 2014, 53 (3), 711-714.

15. Zhang, Y. L.; Zhao, Y.; Yang, B.; Zhu, C. Y.; Wei, Y.; Tao, L., 'One pot' synthesis of well-defined poly(aminophosphonate)s: time for the Kabachnik-Fields reaction on the stage of polymer chemistry. Polym. Chem. 2014, 5 (6), 1857-1862.

16. Kakuchi, R.; Theato, P., Efficient Multicomponent Postpolymerization Modification Based on Kabachnik-Fields Reaction. ACS Macro Lett. 2014, 3 (4), 329-332.

17. Liu, Y. J.; Gao, M.; Lam, J. W. Y.; Hu, R. R.; Tang, B. Z., CopperCatalyzed Polycoupling of Diynes, Primary Amines, and Aldehydes: A New One-Pot Multicomponent Polymerization Tool to Functional Polymers. Macromolecules 2014, 47 (15), 4908-4919.

18. Bachler, P. R.; Schulz, M. D.; Sparks, C. A.; Wagener, K. B.; Sumerlin, B. S., Aminobisphosphonate Polymers via RAFT and a Multicomponent Kabachnik-Fields Reaction. Macromol. Rapid Commun. 2015, 36 (9), 828-833.

19. Theato, P., Multi-Component and Sequential Reactions in Polymer Synthesis. Springer: 2015; Vol. 269.

20. Yang, B.; Zhao, Y.; Wei, Y.; Fu, C. K.; Tao, L., The Ugi reaction in polymer chemistry: syntheses, applications and perspectives. Polym. Chem. 2015, 6 (48), 8233-8239.

21. Zhang, Q. D.; Zhang, Y. L.; Zhao, Y.; Yang, B.; Fu, C. K.; Wei, Y.; Tao, L., Multicomponent Polymerization System Combining Hantzsch Reaction and Reversible Addition-Fragmentation Chain Transfer to Efficiently Synthesize Well-Defined Poly(1,4-dihydropyridine)s. ACS Macro Lett. 2015, 4 (1), 128132.

22. Zhao, Y.; Wu, H. B.; Wang, Z. L.; Wei, Y.; Wang, Z. M.; Tao, L., Training the old dog new tricks: the applications of the Biginelli reaction in polymer chemistry. Sci. China Chem. 2016, 59 (12), 1541-1547.

23. Blasco, E.; Sims, M. B.; Goldmann, A. S.; Sumerlin, B. S.; BarnerKowollik, C., 50th Anniversary Perspective: Polymer Functionalization. Macromolecules 2017, 50 (14), 5215-5252.

24. Llevot, A.; Boukis, A. C.; Oelmann, S.; Wetzel, K.; Meier, M. A. R., An Update on Isocyanide-Based Multicomponent Reactions in Polymer Science. Top. Curr. Chem. 2017, 375 (4), 127-155.
25. Wu, H. B.; Wang, Z. M.; Tao, L., The Hantzsch reaction in polymer chemistry: synthesis and tentative application. Polym. Chem. 2017, 8 (47), 7290-7296.

26. Boukis, A. C.; Reiter, K.; Frolich, M.; Hofheinz, D.; Meier, M. A. R., Multicomponent reactions provide key molecules for secret communication. Nat. Commun. 2018, 9, 1439.

27. Llevot, A.; Boukis, A. C.; Oelmann, S.; Wetzel, K.; Meier, M. A. R., An Update on Isocyanide-Based Multicomponent Reactions in Polymer Science. Top. Curr. Chem. Collec. 2018, 127-155.

28. Li, Y. S.; Yang, L.; Zeng, Y.; Wu, Y. W.; Wei, Y.; Tao, L., SelfHealing Hydrogel with a Double Dynamic Network Comprising Imine and Borate Ester Linkages. Chem. Mater 2019, 31 (15), 5576-5583.

29. Biginelli, P., Ueber Aldehyduramide des Acetessigäthers. Ber. Dtsch. Chem. Ges. 1891, 24 (1), 1317-1319.

30. Kappe, C. O., A reexamination of the mechanism of the Biginelli dihydropyrimidine synthesis. Support for an N-Acyliminium ion intermediate1. J. Org. Chem. 1997, 62 (21), 7201-7204.

31. Matos, L. H. S.; Masson, F. T.; Simeoni, L. A.; Homem-de-Mello, M., Biological activity of dihydropyrimidinone (DHPM) derivatives: A systematic review. Eur. J. Med. Chem. 2018, 143, 1779-1789.

32. Wu, H. B.; Yang, L.; Tao, L., Polymer synthesis by mimicking nature's strategy: the combination of ultra-fast RAFT and the Biginelli reaction. Polym. Chem. 2017, 8 (37), 5679-5687.

33. Mao, T. F.; Liu, G. Q.; Wu, H. B.; Wei, Y.; Gou, Y. Z.; Wang, J.; Tao, L., High Throughput Preparation of UV-Protective Polymers from Essential Oil Extracts via the Biginelli Reaction. J. Am. Chem. Soc. 2018, 140 (22), 6865-6872.

34. Mao, T. F.; Yang, L.; Liu, G. Q.; Wei, Y.; Gou, Y. Z.; Wang, J.; Tao, L., Ferrocene-Containing Polymer via the Biginelli Reaction for In Vivo Treatment of Oxidative Stress Damage. ACS Macro Lett. 2019, 8 (6), 639-645.

35. Yang, L.; Zeng, Y.; Wu, H.; Zhou, C.; Tao, L., An antioxidant selfhealing hydrogel for 3D cell cultures. J. Mater. Chem. B 2020, 8 (7), 1383-1388.

36. Mayer, T. U.; Kapoor, T. M.; Haggarty, S. J.; King, R. W.; Schreiber, S. L.; Mitchison, T. J., Small molecule inhibitor of mitotic spindle bipolarity identified in a phenotype-based screen. Science 1999, 286 (5441), 971-974. 


\section{Anti-cancer Polymers via the Biginelli Reaction}

Yongsan Li, Tianhao Tan, Yuan Zhao, Yen Wei, Dong Wang, Rongjun Chen and Lei Tao

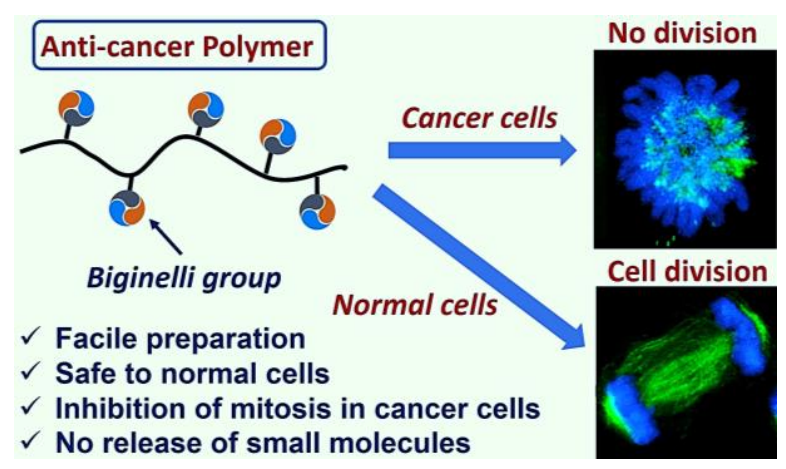

